\title{
Use of a combined SVD-Kalman filter approach for induction motor broken rotor bars identification
}

\author{
K. Azouzi, A. H. Boudinar, F. A.Aimer, A. Bendiabdellah \\ Electrical Engineering Faculty, Diagnostic Group University of Sciences and Technology of Oran, Algeria \\ azouzi_kd@yahoo.fr,boud_ah@yahoo.fr,fethi.aimer@yahoo.fr,bendiazz@yahoo.fr
}

\begin{abstract}
This paper describes a new parametric spectral estimator for the identification of rotor bar fault of an induction motor by analyzing the stator current. This approach combines two methods: The first one, the Singular Value Decomposition method which allows the accurate detection and location of the fault's signature frequency. The second method allows the estimation of the fault amplitude. To this end, the Kalman filter is used for its efficient estimation of both amplitude and phase using the frequencies obtained by the first method. This combination of both methods gives a better frequency resolution for a very short acquisition time, which cannot be obtained using the conventional method of the Periodogram. Moreover, in order to reduce the significant computation time resulting from the use of the Kalman filter, the proposed approach is applied only to the frequency band where the fault signature is likely to appear. A series of tests will be carried out on real signals representing rotor faults.
\end{abstract}

Index Terms - Induction motor, Motor current signature analysis (MCSA), Broken bar, SVD, Kalman filter.

\section{INTRODUCTION}

The induction motor is the most used electric machine in the industry. Its main advantage is the absence of sliding electrical contacts, which leads to a simple and robust structure easy to build with low cost [1]. However, due to certain electrical or mechanical stresses, different faults can occur in the induction motor making the process of detecting these faults necessary to avoid the complete shutdown of the industrial process [2]. Among these faults, broken rotor bar (BRB) faults account for about $20 \%$ of the failures of the medium and high power motors [3]. The consequences of BRB can be various such as the increase in motor vibration, the decrease of starting performances, the fluctuations in load torque or the increase in temperature in the broken zone and in the stator windings [4]. The reason for which, it is necessary to diagnose the incipient rotor's faults.

Among the signal processing methods allowing the detection of BRB faults, we can find the estimation of the Power Spectral Density (PSD) using the Periodogram the technique [5], this method is the most used in industry. Unfortunately, it does not give reliable results when:

$>$ The speed or the load is not constant, which leads to the problem of non-stationary signals [6].

$>$ The acquisition time is short: the problem of frequency resolution [2]. 
In order to overcome the problem of non-stationary signals, several time-frequency and time-scale methods have been proposed to extract information on the variation of signal's frequencies as a function of time. The most well-known are the Short-Time Fourier Transform (STFT), the WignerVille Distribution (WVD) or the Wavelet Transform (WT). The principle of the STFT method consists in analysing the measured signal, by applying the Fourier Transform on short and consecutive temporal segments in which the signal is assumed as stationary. Each segment is analyzed by multiplying it by a weighting window [7]. However, the choice of a window with fixed size causes a serious problem of frequency resolution. Indeed, the choice of a large weighting window to obtain a better frequency resolution causes the loss of a great part of information related to time. Moreover, the choice of a narrow window to ensure a high temporal resolution leads to a low-frequency resolution. This is the principle of the Heisenberg-Gabor uncertainty [8]. To avoid this problem, the WVD and its variants have been proposed. This transformation allows visualizing the signal in timefrequency plan and can be interpreted as a distribution of the energy as a function of the time and frequency. The WVD improves the time-frequency resolution, to the detriment of the aspect of the interference terms in the frequencies due mainly to the noise contained in the signal [9].

To solve all these problems, the WT and its variants have been widely used in large domains of fault diagnosis of electrical machines [9]. This method is based on the use of short duration windows for the high frequencies and long duration windows for the low frequencies, which allows having a multi-resolution analysis. But, this method depends strongly on the analyzing wavelet, and it is also subject to the Heisenberg inequality.

Concerning the second problem of the conventional Periodogram method; linked to the acquisition time which affects the reliability of identification of harmonics very close to each other; several highperformance analysis methods have been developed. These methods are generally based on a mathematical model of the signals to be analysed. Thus, the PSD is estimated [10] from the parameters of this model. These methods are generally called high resolution methods and are divided into two categories [11]: linear prediction methods and subspace techniques. The origin of linear prediction methods goes back to the Prony work [12], aimed at estimating a sum of exponentials by the techniques of linear prediction. Otherwise, the subspace methods are based on the particular properties of the covariance matrix of the acquired signal. So, the study of its rank allows separating the data space into two subspaces, the signal subspace generated by the sinusoids to be identified and the noise subspace which is it's complementary orthogonal. For example, the MUltiple SIgnal Classification (MUSIC) method [13] and its Root-MUSIC variant are based on the noise subspace, and the Estimation of Signal Parameters using Rotational Invariance Techniques (ESPRIT) method [14] is based on the signal subspace. The applications of these methods for the electrical machine faults diagnosis are detailed in [15], [16]. It should be noted finally, that the principal limitations of these methods are due to their low robustness to the unknown signal components number [15] and the unreliable estimation of the amplitudes and phases of the sought harmonics [17]. In addition, these Brazilian Microwave and Optoelectronics Society-SBMO received 26 Oct 2017; for review 26 Oct 2017 ; accepted 23 Feb 2018 Brazilian Society of Electromagnetism-SBMag 
methods require a significant computation time.

The aim of this paper is to study another class of parametric estimators based on a stator current state model. From this modelling, recursive estimation techniques such as the Kalman filter (KF) are combined with the Singular Value Decomposition (SVD) of the covariance matrix of the stator current to identify the frequencies and to estimate the corresponding amplitudes characterizing the BRB faults in a direct way. In fact, numerous studies [18], [19] have shown the efficiency of the KF to solve many practical problems in various domains related to signal processing namely radar detection, image processing and industrial machinery control.

In the electrical machines diagnosis field, the KF is generally used to estimate parameters and inaccessible quantities such as rotor current, rotor flux and rotor bars resistance [20], [21]. Moreover, we have opted for the Kalman estimator as a new approach because it proves its performances in amplitudes estimation even in the presence of strongly noisy signals. Indeed, it is very difficult to estimate the amplitudes of the incipient faults harmonics in noisy signals.

In addition, the SVD is used to separate the signal subspace from the noise subspace. So, the frequencies of the searched harmonics are determined from the subspace signal.

Thus, in this article, the KF is used to estimate the amplitudes and phases of the sought harmonics faults by exploiting the frequencies obtained by SVD.

Nevertheless, this approach requires an important computation time, which can be a serious handicap for its implementation in real time. However, since the frequency signature of a fault is located on a known frequency band [15], then the proposed solution in this paper consists of the decrease of the computation time and the used memory space by applying the processing only on the frequency band where the signature of the fault is likely to appear. To highlight the contribution merits of this new approach in the detection and the monitoring of rotor faults severity, several experimental tests are conducted on an induction motor by analysing the stator current signal in the steady-state and without varying the load or speed of the motor.

\section{STATOR CURRENT SIGNATURE ANALYSIS}

The BRB fault causes an imbalance in the rotor currents, it results, therefore, the appearance of additional harmonics in the spectral content of the stator current. Indeed, the broken rotor bars fault is manifested by the presence of harmonic components around the fundamental corresponding to the frequencies given by the following relation [2], [22]:

$$
f_{b b}=(1 \pm 2 k s) f_{s} \quad \text { With } \quad k=1,2,3, \ldots
$$

Where $f_{b b}$ are the sideband frequencies associated with the broken rotor bar, $f_{s}$ the supply frequency and $s$ is the slip of the motor.

The harmonics appearing on the lower sideband $f_{b b}=(1-2 k s) f_{s}$ are due to the effect of the BRB, while the harmonics appearing on the upper sideband $f_{b b}=(1+2 k s) f_{s}$ are due to variations in speed. 
The amplitudes of these lateral bands are proportional to the number of broken bars and the motor load [23].

\section{PRINCIPLE OF THE PROPOSED METHOD}

In this paper, the proposed approach is based on the use of KF as a tool for estimating the frequencies signatures of sought faults. Note those two filters types can be used, the Linear Kalman Filter (LKF) and the Extended Kalman Filter (EKF). The EKF is very efficient as an estimator but tends to deviate from the limits of the linearity zone during the estimation of the sought parameters.

In order to avoid this disadvantage, this paper proposes to decompose the problem of signal parameters estimation (amplitudes, frequencies, and phases) using two distinct estimators. The first aims in decomposing the covariance matrix of the stator current into singular values (SVD) in order to determine the sought frequencies. The second, which is the KF, allows estimating the amplitudes and phases of sought harmonics. Thus, the problem of no linearity will be avoided. This procedure is illustrated in figure 1.

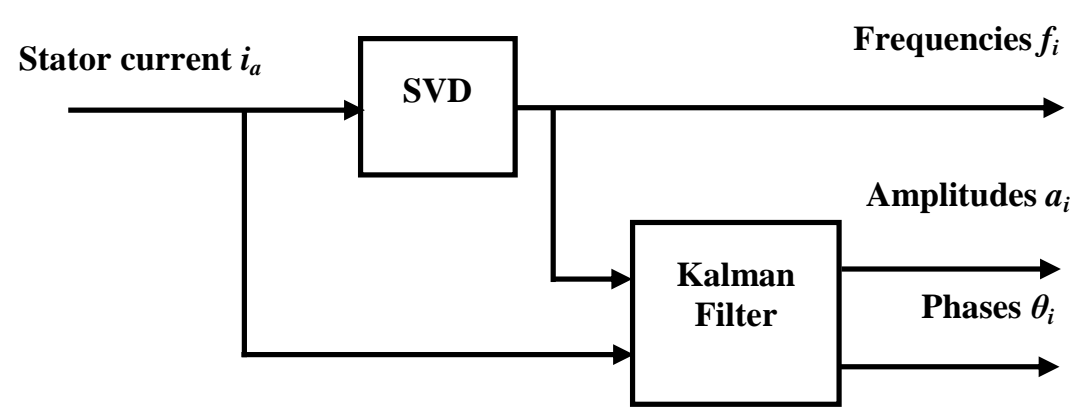

Fig.1. Principle of the proposed method

\section{A. Data model}

According to several studies [11], [24], in the presence of electrical or mechanical faults, the stator current model of the three phase induction motor in discrete-time is expressed by:

$$
i_{a}(n)=\sum_{i=1}^{L} a_{i} \sin \left(2 \pi f_{i} \frac{n}{F_{s p}}+\theta_{i}\right)+b(n) \quad ; n=0,1, \ldots, N-1
$$

Where $N$ is the number of the acquired samples, $a_{i}, f_{i}$ and $\theta_{i}$ are respectively the amplitude, the frequency and the phase of the $i^{\text {th }}$ sinusoidal component, $F_{s p}$ is the sampling frequency, $b(n)$ a measurements noise that can be modelled as an additive white noise and $L$ the number of sinusoidal components.

From Euler's decomposition, equation (2) can be written as follows

$$
i_{a}(n)=\sum_{i=1}^{2 L} \frac{a_{i} e^{j \theta_{i}}}{2 j} e^{j 2 \pi \frac{f_{i}}{F_{s p}} n}+b(n)=\sum_{i=1}^{2 L} \mathrm{~A}_{i} e^{j 2 \pi f_{i} \frac{n}{F_{s p}}}+b(n)
$$

Where $\mathrm{A}_{\mathrm{i}}$ represents the complex amplitudes

In matrix form, equation (3) is written [11]: 


$$
I_{a}=S A+B
$$

Where $I_{a}, A$, and $B$ represent respectively the vectors of observation (stator current), the amplitude, and the noise. These are defined as follows:

$$
\begin{gathered}
I_{a}=\left[i_{a}(0) \ldots i_{a}(N-1)\right]^{T} \\
A=\left[\mathrm{A}_{1} \mathrm{~A}_{2} \ldots \mathrm{A}_{2 L}\right]^{T} \\
B=[b(0) \ldots b(N-1)]^{T}
\end{gathered}
$$

Where $S$ represents the Vandermonde matrix:

$$
S=\left[s_{1} s_{2} \ldots s_{k} \ldots s_{2 L}\right]
$$

With

$$
s_{k}=\left[1 e^{j 2 \pi f_{k} \frac{1}{F_{\text {sp }}}} e^{j 2 \pi f_{k} \frac{2}{F_{\text {sp }}}} \ldots e^{j 2 \pi \pi_{k} \frac{(N-1)}{F_{\text {sp }}}}\right]^{T}
$$

From the data model defined by Equation 4, the desired frequencies based on the first part of our approach can be determined using the SVD method.

\section{B. Estimation of frequencies by the SVD method}

The estimation of frequencies is based on the SVD of the covariance matrix of the noisy signal $i_{a}(n)$ to be analysed. This matrix is defined by the following relation:

$$
Y_{a}=\left\{I_{a} I_{a}^{H}\right\}
$$

Where $\mathrm{E}\{$.$\} denotes the mathematical expectation and (. )^{\mathrm{H}}$ is the Hermitian transposition operator.

Moreover, this matrix can be considered as the sum of two matrices of covariance that of the signal and the other related to the noise.

$$
Y_{a}=E\left\{I_{a} I_{a}^{H}\right\}=Y_{s}+Y_{B}=S \Psi S^{H}+\sigma^{2} I
$$

Where $\sigma^{2}$ is the variance of white noise and $\Psi$ is the power matrix of the harmonics defined as:

$$
\Psi=\operatorname{diag}\left[A_{1}^{2} A_{2}^{2} \cdots A_{2 L}^{2}\right]
$$

The decomposition in SVD allows the separation of the matrix $Y_{\mathrm{a}}$ of dimension $N \times N$ in several orthogonal components according to the classification of these singular values [25]:

$$
Y_{a}=\sum_{i=1}^{2 L} \lambda_{i} g_{i} u_{i}^{H}+\sum_{i=2 L+1}^{N} \lambda_{i} g_{i} u_{i}^{H}
$$

In matrix form the previous equation is written:

$$
Y_{a}=\left[\begin{array}{ll}
G_{1} & G_{2}
\end{array}\right]\left[\begin{array}{cc}
\Sigma_{1} & 0 \\
0 & \Sigma_{2}
\end{array}\right]\left[\begin{array}{c}
U_{1}^{H} \\
U_{2}^{H}
\end{array}\right]
$$

Where

$$
\begin{aligned}
& G_{1}=\left[g_{1}, g_{2}, \ldots, g_{2 L}\right], G_{2}=\left[g_{2 L+1}, g_{2 L+2}, \ldots, g_{N}\right] \\
& U_{1}=\left[u_{1}, u_{2}, \ldots ., u_{2 L}\right], U_{2}=\left[u_{2 L+1}, u_{2 L+2}, \ldots . ., u_{N}\right]
\end{aligned}
$$


The matrix $\left[G_{l} G_{2}\right]$ is composed by the $N$ left singular vectors (eigenvectors of $Y_{a} Y_{a}^{H}$ ). The matrix $\left[\begin{array}{ll}U_{1} & U 2\end{array}\right]$ is composed of the $N$ right singular vectors (eigenvector of $Y_{a}^{H} Y_{a}$ ). $\sum_{1}=\operatorname{diag}\left(\lambda_{1}, \lambda_{2}, \ldots . . \lambda_{2 L}\right)$ and $\sum_{2}=\sigma^{2} I_{N-2 L}$. Knowing that the $\lambda_{i}$ are the singular values of $Y_{a}$ ranked in descending order:

$$
\text { This classification in } \underbrace{\lambda_{1} \geq \lambda_{2} \geq \ldots \geq \lambda_{2 L}}_{\text {Espacesignal }}>\underbrace{\lambda_{2 L+1}=\lambda_{2 L+2}=\ldots=\lambda_{N}=\sigma^{2}}_{\text {Espacebruit }}
$$

the descending order of the singular values $\lambda_{i}$ of the matrix $Y_{a}$ allows to distinguish between the signal subspace and the noise subspace. Indeed, the $2 L$ first singular values generate the signal subspace $\left(G_{1}\right.$ and $\left.U_{1}\right)$ and the $N-2 L$ singular values represent the noise subspace $\left(G_{2}\right.$ and $\left.U_{2}\right)$.

To estimate the harmonics frequencies, two matrices $D_{1}$ and $D_{2}$ of the same dimensions $((N-1) \times 2 L)$ can be produced from matrix $G_{l}$ such that:

$$
G_{1}=\left[\begin{array}{c}
D_{1} \\
\cdots
\end{array}\right]=\left[\begin{array}{c}
\cdots \\
D_{2}
\end{array}\right]
$$

With $D_{1}$ the matrix representing the $(N-1)$ first rows of the matrix $G_{l}$ and $D_{2}$ the $(N-1)$ last rows. The two matrices $D_{1}$ and $D_{2}$ are linked by the following relation:

$$
D_{2} \phi=D_{1}
$$

Where

$$
\phi=\operatorname{diag}\left(\varphi_{1}, \varphi_{2}, \ldots \varphi_{i}, \ldots \varphi_{2 L}\right)
$$

And $\quad \varphi_{i}=e^{j 2 \pi \frac{f_{i}}{F_{s p}}}$

Thus, the estimation of the searched frequencies can be deduced from the following relation:

$$
f_{i}=\frac{F_{s p}}{2 \pi} \operatorname{Arg}\left(\varphi_{i}\right)
$$

However, to make the proposed technique SVD-Kalman Filter effective, the matrix $Y_{a}$ must be nonsingular. Unfortunately, this condition is not always verified in practice because in most cases, the estimation of the searched frequencies must be made from only one data vector (a single measure). Under these conditions, the matrix $Y_{a}$ is estimated by the following equation [15]:

$$
\hat{Y}_{a}=\frac{1}{N-M+1} \Phi \Phi^{H}
$$

Where $M$ and $\Phi$ are respectively the order of the data matrix and the Hankel matrix defined by: 


$$
\Phi=\left[\begin{array}{cccc}
i_{a}(0) & i_{a}(1) & \cdots & i_{a}(N-M) \\
i_{a}(1) & i_{a}(2) & \cdots & i_{a}(N-M+1) \\
\vdots & \vdots & \cdots & \vdots \\
i_{a}(M-1) & i_{a}(M) & \cdots & i_{a}(N-1)
\end{array}\right]^{H}
$$

The next step is dedicated to the estimation of both amplitudes and phases.

\section{Estimation of the amplitudes and the phases by the Kalman filter}

The KF is a recursive algorithm used in the construction of optimal linear estimators in the sense that they minimize the mean squared error. Moreover, the KF does not require, unlike the Discrete Fourier Transform (DFT), that the number of samples per period is an integer of the power of 2.

This filter is able to estimate the parameters of a signal observed from a previously defined state model [26] from the steady state mode of the signal. It assumes that the signal can be approximated by a limited number of $L$ harmonic components.

This filter lies in two main equations. The first is the state equation and the second is the measure equation:

$$
\left\{\begin{array}{l}
\underline{X}_{K+1}=F_{K} \underline{X}_{K}+\underline{W}_{K} \\
\underline{Z}_{K}=H_{K} \underline{X}_{K}+\underline{V}_{K}
\end{array} \quad \text { With } \quad k=0,1, \ldots, N-1\right.
$$

Where $\underline{X}_{k}$ is the state vector of the process, $F_{k}$ is the state transition matrix which describes the dynamics of $\underline{X}_{k}$ and $\underline{W}_{k}$ is the model error of the process assumed to be Gaussian white of covariance matrix $Q_{\mathrm{k}}$ and of zero mean.

$\underline{Z}_{k}$ being the measurement vector at time $k, H_{k}$ the observation matrix and $\underline{V}_{\mathrm{k}}$ the measurement error also assumed to be the Gaussian white noise with covariance matrix $R_{k}$ and of zero mean supposed uncorrelated with $\underline{W}_{k}$.

In our case, the measurement vector $\underline{Z}$ represents the stator current modelled according to equation (2) and which can be rewritten in the following form:

$$
Z_{k}=\sum_{i=1}^{L} a_{i} \cos \left(\theta_{i}\right) \sin \left(2 \pi f_{i} k \frac{1}{F_{s p}}\right)+a_{i} \sin \left(\theta_{i}\right) \cos \left(2 \pi f_{i} k \frac{1}{F_{s p}}\right)
$$

This model includes $L$ harmonic components of unknown amplitudes and phases but of frequencies estimated in the previous part.

From Equation (23), it can be assumed that:

$$
\left\{\begin{array}{ccc}
x_{1}=a_{1} \cos \left(\theta_{1}\right) & , & x_{2}=a_{1} \sin \left(\theta_{1}\right) \\
x_{3}=a_{2} \cos \left(\theta_{2}\right) & , & x_{4}=a_{2} \sin \left(\theta_{2}\right) \\
\ldots \ldots \ldots \ldots & \ldots \ldots \ldots \\
x_{2 i-1}=a_{i} \cos \left(\theta_{i}\right), & & x_{2 i}=a_{i} \sin \left(\theta_{i}\right)
\end{array}\right.
$$

The variables $\left[x_{1}, x_{2}, \ldots x_{2 i-1}, x_{2 i}\right]$ for $i=1,2, \ldots \ldots, L$ represent the components in phase and in phase quadrature and are referenced as state variables. This choice leads to define the state equation as Brazilian Microwave and Optoelectronics Society-SBMO received 26 Oct 2017; for review 26 Oct 2017; accepted 23 Feb 2018 
follows:

$$
\left[\begin{array}{l}
x_{1} \\
x_{2} \\
\cdots \\
x_{2 i-1} \\
x_{2 i}
\end{array}\right]_{K+1}=F_{K} \underline{X}_{K}+\underline{W}_{K}=\left[\begin{array}{ccccc}
1 & 0 & 0 & \cdots & 0 \\
0 & 1 & 0 & \cdots & 0 \\
0 & 0 & 1 & \cdots & 0 \\
\vdots & \vdots & \vdots & \ddots & \vdots \\
0 & 0 & 0 & \cdots & 1
\end{array}\right]\left[\begin{array}{l}
x_{1} \\
x_{2} \\
\cdots \\
x_{2 i-1} \\
x_{2 i}
\end{array}\right]_{K}+\underline{W}_{K}
$$

Also, considering equation (24), the measurement equation becomes:

$$
Z_{k}=\sum_{i=1}^{L} x_{2 i-1} \sin \left(2 \pi f_{i} k \frac{1}{F_{s p}}\right)+x_{2 i} \cos \left(2 \pi f_{i} k \frac{1}{F_{s p}}\right)
$$

This allows rewriting this equation as follows:

$$
\underline{Z}_{K}=H_{K} \underline{X}_{K}+\underline{V}_{K}=\left[\begin{array}{c}
\sin \left(2 \pi f_{1} k \frac{1}{F_{s p}}\right) \\
\cos \left(2 \pi f_{1} k \frac{1}{F_{s p}}\right) \\
\cdots \\
\sin \left(2 \pi f_{L} k \frac{1}{F_{s p}}\right) \\
\cos \left(2 \pi f_{L} k \frac{1}{F_{s p}}\right)
\end{array}\right]^{T}\left[\begin{array}{l}
x_{1} \\
x_{2} \\
\cdots \\
x_{2 i-1} \\
x_{2 i}
\end{array}\right]_{K}+\underline{V}_{K}
$$

Under these conditions, the observation matrix can be defined as follows:

$$
H_{k}=\left[\sin \left(2 \pi f_{1} k \frac{1}{F_{s p}}\right) \cos \left(2 \pi f_{1} k \frac{1}{F_{s p}}\right) \ldots . \sin \left(2 \pi f_{L} k \frac{1}{F_{s p}}\right) \cos \left(2 \pi f_{L} k \frac{1}{F_{s p}}\right)\right]
$$

It should be noted that the frequencies $\left[f_{1} f_{2} \ldots \ldots f_{L}\right]$ mentioned in $H_{k}$, are already estimated. This allows us to make the KF model described above as linear. The choice of a linear model is motivated by considerations such as algorithmic simplicity, convergence speed and stability of the estimation in the steady-state mode. Thus, from the state variables of equation (24), the amplitudes of the harmonic components can be estimated by the following expression:

$$
a_{i}=\sqrt{x_{2 i-1}^{2}+x_{2 i}^{2}}
$$

Similarly, for the phases we have:

$$
\theta_{i}=\operatorname{Arg}\left(\frac{x_{2 i}}{x_{2 i-1}}\right) \quad ; i=1,2, \cdots, L
$$

In conclusion, the computation steps for estimating both amplitudes and phases of the harmonics using the discrete Kalman filter are [26]:

The prediction step:

1. State vector estimation: 


$$
\hat{X}_{k+1 / k}=F_{k} \hat{X}_{k / k}
$$

2. Covariance matrix computation of the prediction error:

$$
P_{k+1 / k}=F_{k} P_{k / k} F_{k}^{T}+Q_{k}
$$

The correction step:

3. Computation of the Kalman Gain:

$$
K_{k+1}=P_{k+1 / k} H_{k+1}^{T}\left[H_{k+1} P_{k+1 / k} H_{k+1}^{T}+R_{k+1}\right]^{-1}
$$

4. Estimation of the state vector (update):

$$
\hat{X}_{k+1 / k+1}=\hat{X}_{k+1 / k}+K_{k+1}\left(Z_{k+1}-Z_{k+1 / k}^{\wedge}\right)
$$

5. Computation of the covariance matrix of the error (update):

$$
P_{k+1 / k+1}=\left(1-K_{k+1} H_{k+1}\right) P_{k+1 / k}
$$

\section{Proposed Improvements}

The major drawback of the SVD-Kalman Filter method is the important computation time due to the singular values calculation operation mainly because of the amplitudes estimation by the Kalman filter. This computation time increases exponentially with the number of searched harmonics and the number of used samples. However, it is well known, that the signature of the broken rotor bars fault appears on a limited frequency band $\left[f_{\min }, f_{\max }\right]$ located around the fundamental harmonic. The suggested idea consists, therefore, to apply the proposed technique SVD-Kalman Filter only on this frequency band and not on the entire spectrum of the stator current. This solution will significantly reduce the length of the signal to be analysed and therefore the computation time [15].

With this solution, the processing will thus be performed on $N_{b}=2 \cdot N \cdot f_{\text {Band }} / F_{s p}$, instead of the $N$ starting samples. $N_{b}$ is the number of samples with $f_{\text {Band }}=f_{\max }-f_{\min }$. This solution is illustrated in Fig. 2. 

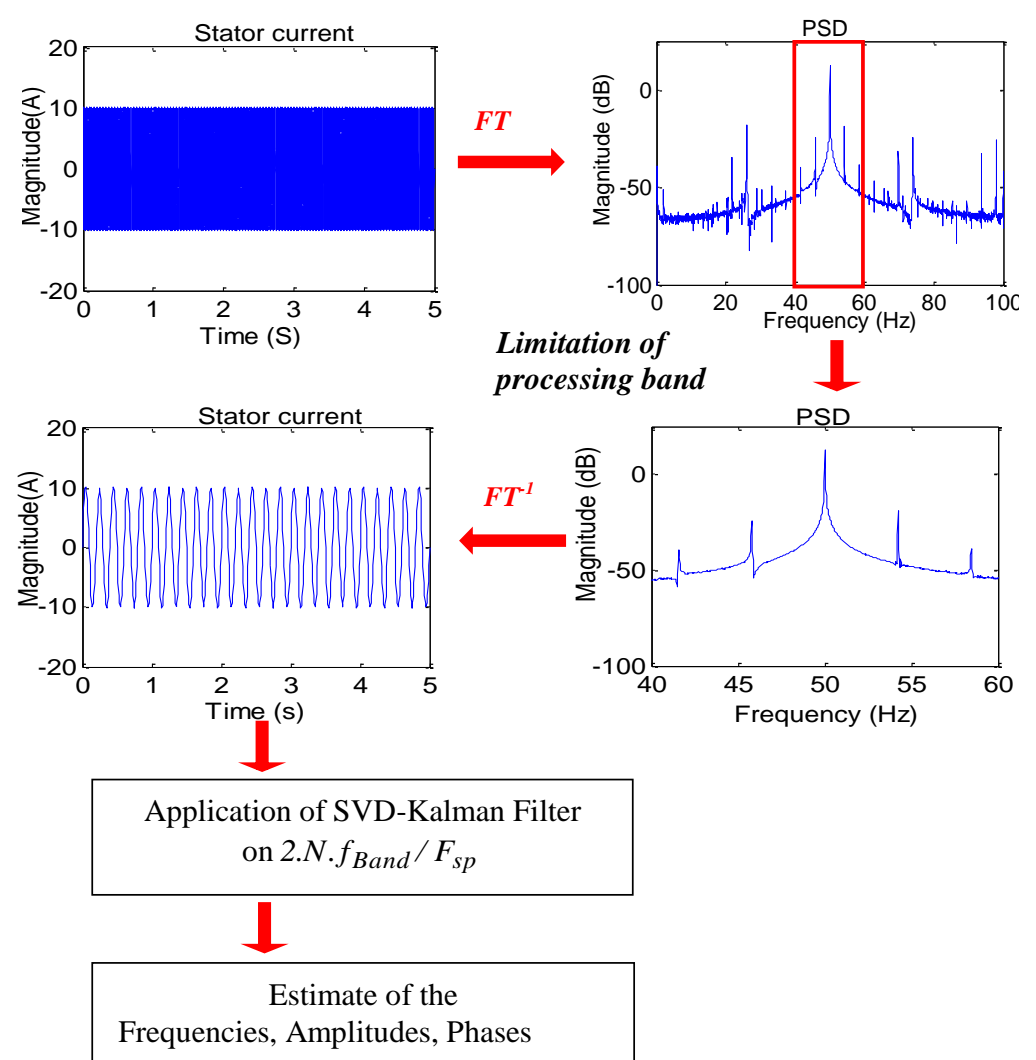

Fig. 2. Proposed solution for the reduction of the computation time $F T$ : The Fourier transforms, $F T^{-1}$ : The inverse Fourier transform

\section{EXPERIMENTAL RESULTS}

The experimental setup used in this study consists of:

1. A three-phase squirrel cage induction motor: ENEL, $50 \mathrm{~Hz}, 4$ poles, $3 \mathrm{~kW}, 7 \mathrm{~A}$ and $1410 \mathrm{rpm}$. The rotors used are composed of 28 bars which can be interchanged according to the number of the broken bar.

2. A DC machine 200V/10.9A with separate excitation used as a generator. This machine is coupled to the induction motor and debits on a variable resistive load. This allows varying the load torque.

3. A measuring system includes two current Hall Effect sensors (Fluck i30s), an antialiasing filter (realized in our laboratory) with a $400-\mathrm{Hz}$ adjustable cut off frequency chosen for our tests, and an acquisition card (NI-6330). The whole set is connected to a computer for viewing and processing of acquired signals as shown in Fig. 3.

To note that all acquisitions were performed at a constant speed $1440 \mathrm{r} / \mathrm{min}$ in steady state on an acquisition time of $40 \mathrm{~s}$ and with a sampling frequency of $3 \mathrm{KHz}$. In these conditions, the signal length is equal to 120000 samples and the frequency resolution is equal to $0.025 \mathrm{~Hz}$. 


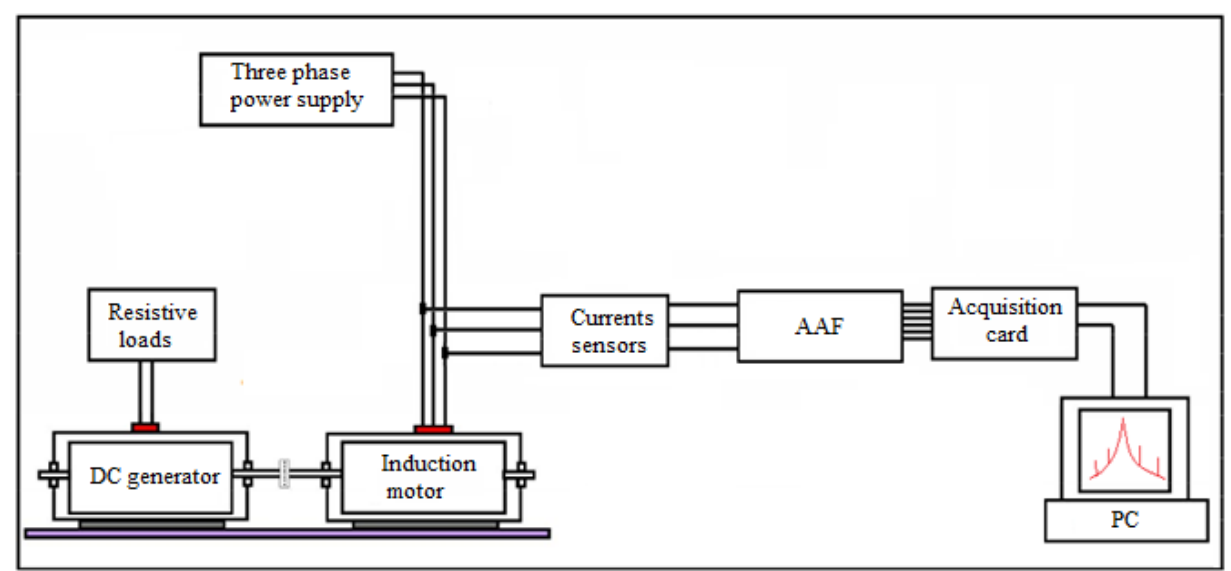

(a)

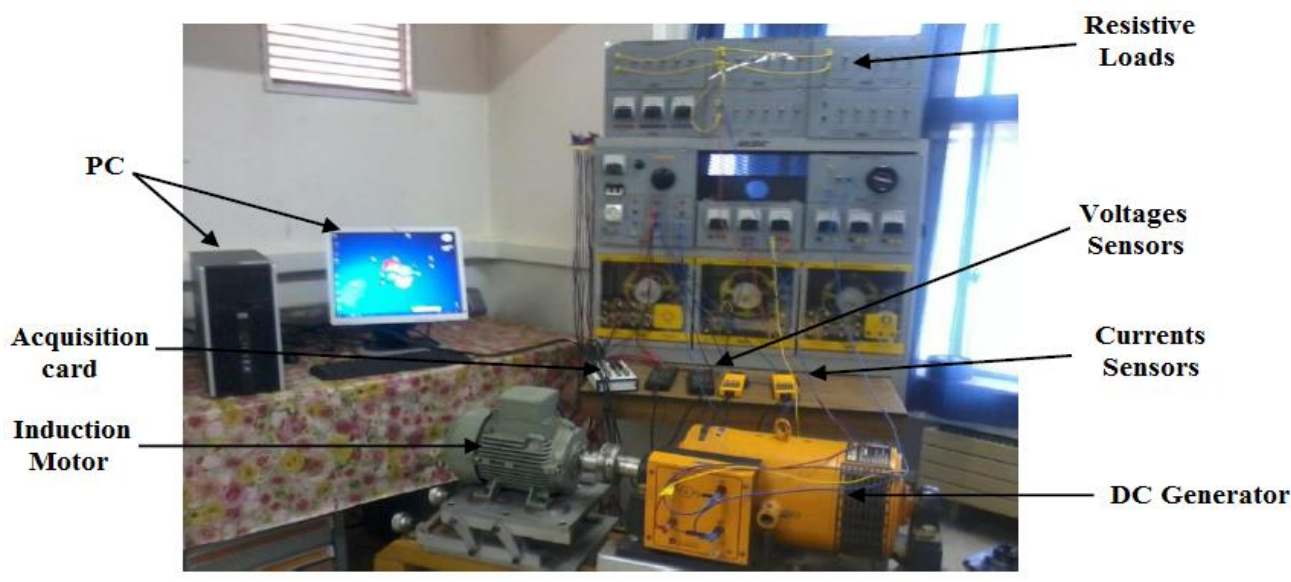

(b)

Fig.3. Realised test rig

The BRB faults are artificially created by drilling the rotor bars. The holes dimensions are $4 \mathrm{~mm}$ in diameter. Figures $4 \mathrm{a}$ and $4 \mathrm{~b}$ illustrate the created faults in rotor bars used in our experimental tests.

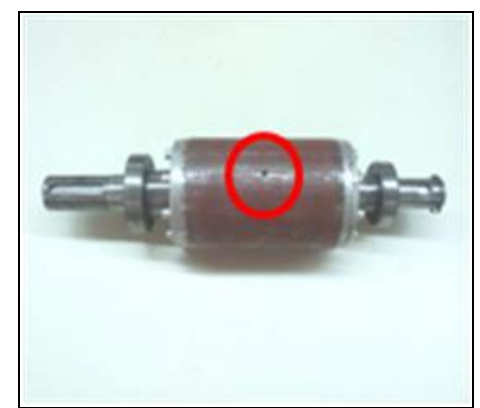

(a)

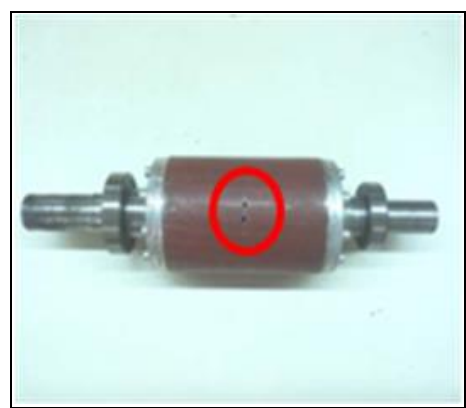

(b)

Fig. 4. Photo of a squirrel cage rotor with broken bars faults; (a) One broken bar, (b) Two broken bars

To note that all acquisitions were performed at a constant speed $1440 \mathrm{r} / \mathrm{min}$ in steady state on an acquisition time of 40s and with a sampling frequency of $3 \mathrm{KHz}$. In these conditions, the signal length is equal to 120000 samples and the frequency resolution is equal to $0.025 \mathrm{~Hz}$. 
The various modes of operation investigated in order to validate our diagnostic approach are:

Operation of the motor with a healthy rotor

Operation of the motor with a one broken bar

Operation of the motor with two broken rotor bars

Moreover, according to equation (1), the frequency signature of BRB fault must appear at the following theoretical frequencies given in Table I.

TABLE I. THEORETICAL ROTOR FAULT FREQUENCIES AT 1440 RPM

\begin{tabular}{lllllc}
\hline \multirow{2}{*}{$f_{b}=(1 \pm 2 k s) f_{s}$} & \multicolumn{2}{c}{ Lower Sideband } & & \multicolumn{2}{c}{ Upper Sideband } \\
\cline { 2 - 3 } & $\boldsymbol{k}=\mathbf{k}=\mathbf{1}$ & $f_{s}$ & & $\boldsymbol{k}=\mathbf{2}$ \\
\hline Frequencies (Hz) & 42 & 46 & 50 & 54 & 58 \\
\hline
\end{tabular}

This table shows that an analysis of the stator current spectrum around the fundamental over a frequency band of $[40 \mathrm{~Hz}, 60 \mathrm{~Hz}]$ is sufficient for the diagnosis of the rotor faults for all the operation modes cited previously. Moreover, the choice of this frequency range is justified by the fact that the slip of induction motors can never exceed $7 \%$ in reality even when it is overloaded [2].

\section{A. Motor operation with healthy rotor}

In this first test, the motor is tested with a rotor supposed to be healthy. It is to be noted that, what we call a healthy rotor in this paper, is a rotor that has no visually apparent faults. This does not exclude the existence of imperfections linked either to the phase of manufacture or to the existence of scratches associated with its use. This first mode of operation is very important, in order to have the reference signals. Indeed, the signals acquired and analysed for this first test will be used as references for all future tests. This step is called the "reference step".

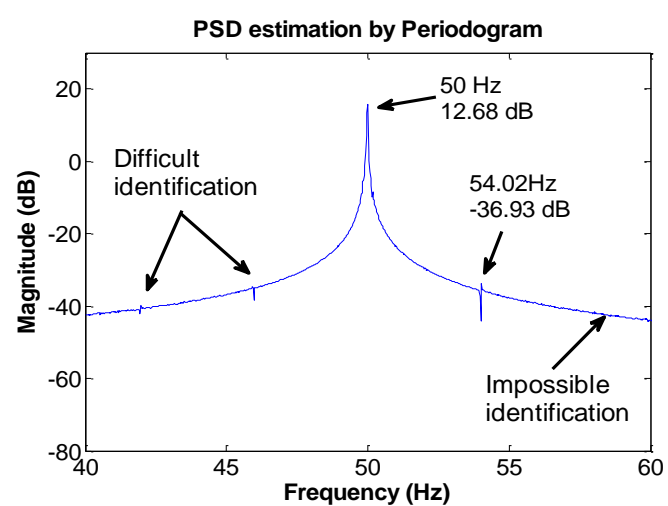

(a)

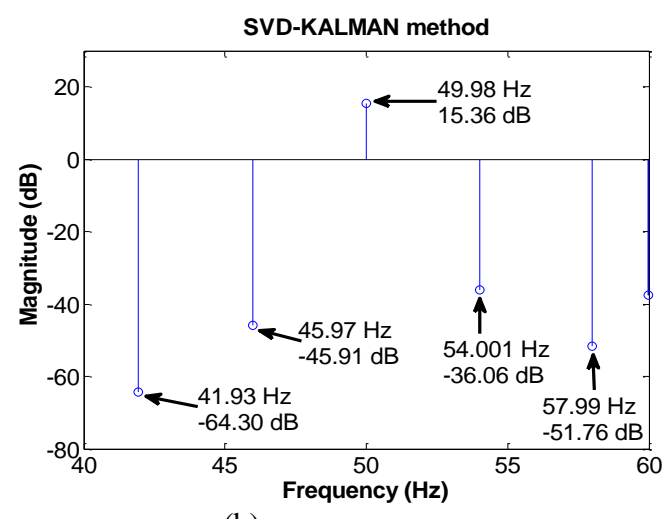

(b)

Fig.5. Stator current analysis Healthy rotor; (a) PSD method, (b) SVD-KALMAN method

Figure $5 a$ shows the results obtained by the conventional method based on the PSD estimation (by the Periodogram). This figure depicts the appearance of a low harmonic at the frequency of $54.02 \mathrm{~Hz}$ in addition to the fundamental $\left(f_{\mathrm{s}}=50 \mathrm{~Hz}\right)$. On the other hand, in the lower sideband, the identification of other harmonics is difficult or even impossible. Under these conditions, it is difficult to explain the presence of this frequency at $54.02 \mathrm{~Hz}$.

By analysing the stator current with the SVD-Kalman Filter method in the specified frequency band Brazilian Microwave and Optoelectronics Society-SBMO received 26 Oct 2017; for review 26 Oct 2017; accepted 23 Feb 2018 
[40Hz, $60 \mathrm{~Hz}]$, see Fig. $5 b$, the following harmonics summarized in Table II are obtained.

TABLE II. HARMONICS ESTIMATION BY THE SVD-KALMAN METHOD FOR THE FIRST OPERATING MODE ON THE FREQUENCY BAND [40HZ -60HZ] (HEALTHY ROTOR)

\begin{tabular}{|c|c|c|c|c|c|c|}
\hline & & \multicolumn{3}{|c|}{ Lower Sideband } & \multicolumn{2}{|c|}{ Upper Sideband } \\
\hline & & $k=2$ & $k=1$ & $f s$ & $k=1$ & $k=2$ \\
\hline \multicolumn{2}{|c|}{ Estimated frequencies $(\mathrm{Hz})$} & 41.93 & 45.97 & 49.98 & 54.001 & 57.99 \\
\hline \multirow{2}{*}{ Estimated Amplitudes } & $(\mathrm{dB})$ & -64.30 & -45.91 & 15.36 & -36.06 & -51.76 \\
\hline & (A) & 0.0009 & 0.0072 & 8.296 & 0.0222 & 0.0037 \\
\hline
\end{tabular}

The analysis of the harmonics frequency positions generated around the fundamental and their comparison with the given theoretical frequencies in Table I prove the presence of a signature which can be justified by the presence of a rotor cage imbalance or a rotor scratch resulting from the multiple manipulations during the various experimental tests in our laboratory.

These first results show the effectiveness of the proposed method in comparison to the conventional Periodogram method for the detection of very low magnitude harmonics.

\section{B. Effect of the proposed solution}

In the aim to make the proposed method more competitive for a possible use in real-time, the reduction of computation time is necessary. The proposed solution consists to only analyse the frequency band where the signature of the fault can appear. It is to be noted that due to the chosen frequency band $[40 \mathrm{~Hz}, 60 \mathrm{~Hz}]$, the computation time has been improved as it can be shown in Table III.

TABLE III. ESTIMATION OF COMPUTATION TIME

\begin{tabular}{lcc}
\hline Method & Data length & Computation time (s) \\
\hline Periodogram & 120000 & 0.023 \\
\hline SVD-Kalman & 120000 & 40971.86 \\
\hline $\begin{array}{l}\text { Improved SVD-Kalman } \\
\text { On [40Hz 60 Hz] }\end{array}$ & 1600 & 0.40 \\
\hline
\end{tabular}

Table III gives an estimation of the computation time by the mentioned methods for the identification of the components of the fundamental and the searched harmonics. These results are obtained from a microcomputer type Intel Core I3 CPU $2.10 \mathrm{GHz}, 4$ GB RAM.

It can be noticed that the computation time obtained by the Periodogram method is very short compared to the proposed method hence its main advantage. Indeed, the SVD-Kalman method requires a significant time of computation in the order of $40971.86 \mathrm{sec}$, which is approximately 11 hours since it requires analysis of the whole spectrum (that is a large number of samples) which affects the rank of the covariance matrix. This computation time can be a serious problem while implementing the technique in real time.

To deal with this problem, the SVD-Kalman technique is only applied in the frequency band $[40 \mathrm{~Hz}$ 
$60 \mathrm{~Hz}$, which reduces the computation time to $0.40 \mathrm{sec}$. Note that the computation time depends on the samples number used and on the searched harmonics number.

\section{Motor operation with one broken rotor bar}

Figure $6 a$ below represents the stator current spectrum obtained by the conventional method of the PSD estimation using the Periodogram algorithm.

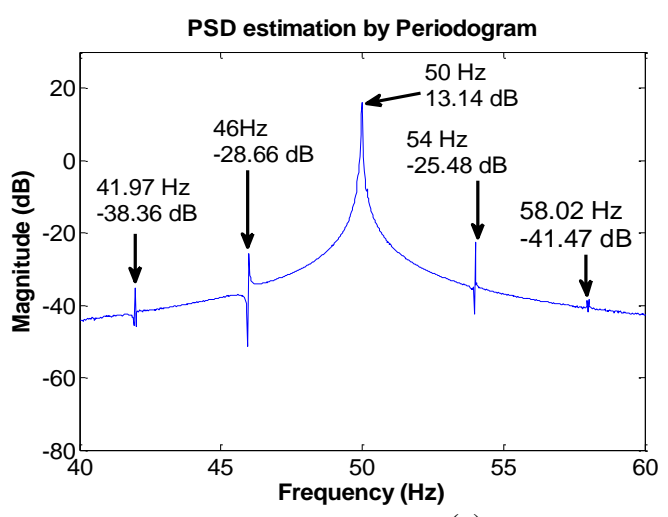

(a)

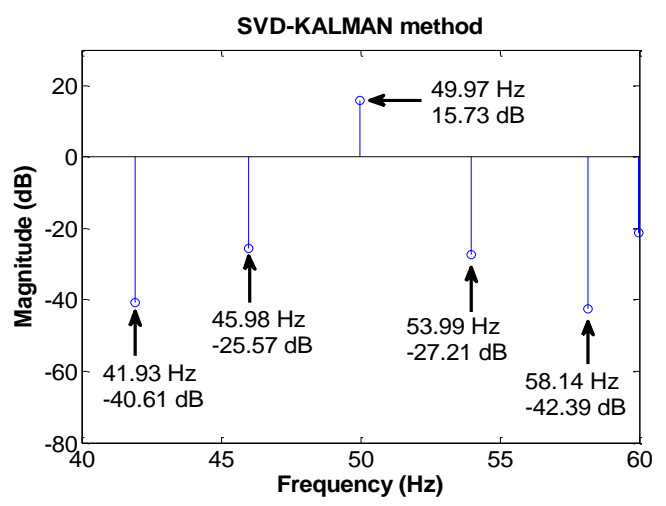

(b)

Fig. 6. Stator current analysis One broken rotor bar; (a) PSD method, (b) SVD-KALMAN method

The stator current analysis for this operation mode by the SVD-Kalman method in the frequency band $[40 \mathrm{~Hz} 60 \mathrm{~Hz}]$ gives the harmonics summarized in the following Table IV:

TABLE IV. HARMONICS ESTIMATION BY IMPROVED SVD-KALMAN METHOD FOR THE CASE OF A ONE BROKEN BAR ON THE FREQUENTIAL BAND $[40 \mathrm{~Hz}, 60 \mathrm{~Hz}]$

\begin{tabular}{lllllll}
\hline & \multicolumn{2}{c}{ Lower Sideband } & & \multicolumn{2}{c}{ Upper Sideband } \\
& \multicolumn{1}{c}{$\boldsymbol{k}=\mathbf{2}$} & $\boldsymbol{k}=\mathbf{1}$ & & $\boldsymbol{f}$ s & $\boldsymbol{k}=\mathbf{1}$ & $\boldsymbol{k}=\mathbf{2}$ \\
\hline \multirow{2}{*}{ Estimated frequencies (Hz) } & 41.93 & 45.98 & 49.98 & 53.99 & 58.14 \\
\hline \multirow{2}{*}{ Estimated Amplitudes } & $(\mathrm{dB})$ & -40.61 & -25.57 & 15.73 & -27.21 & -42.39 \\
\cline { 2 - 7 } & (A) & 0.0132 & 0.0744 & 8.6545 & 0.0616 & 0.0107 \\
\hline
\end{tabular}

Figure $6 b$ shows the spectral clarity given by the proposed SVD-Kalman method. Moreover, we can notice that the harmonics magnitudes around the fundamental, in the case of one broken rotor bar, are higher compared to those of the healthy rotor.

It is to be noted that this slight difference between theoretical and estimated frequency values is certainly due to the error in measuring the mechanical speed of the motor.

\section{Motor Operation With Two Broken Rotor Bars}

Figure $7 a$ illustrates the PSD obtained by the stator current Periodogram. It can be noticed that the harmonics obtained are almost at the same frequencies because the operation mode is always at nominal load. On the other hand, their magnitudes are higher, which indicates that the severity of the fault has increased. 


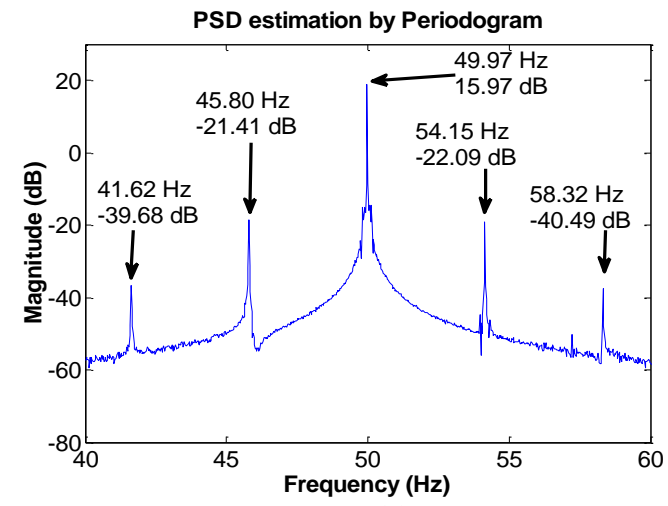

(a)

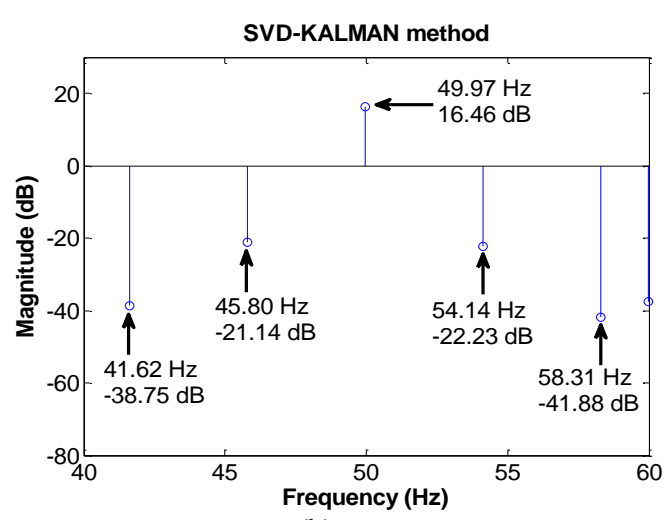

(b)

Fig.7. Stator current analysis Two broken rotor bars; (a) PSD method, (b) SVD-KALMAN method

Furthermore, the identification of the fault frequency signature by the proposed technique SVDKalman is given by the following table:

TABLE V. HARMONICS ESTIMATION BY IMPROVED SVD-KALMAN METHOD FOR THE CASE OF A TOW BROKEN BAR ON THE FREQUENTIAL BAND [40HZ, 60HZ]

\begin{tabular}{lllllll}
\hline & & \multicolumn{2}{c}{ Lower Sideband } & \multicolumn{2}{c}{ Upper Sideband } \\
& & $\boldsymbol{k}=\mathbf{2}$ & $\boldsymbol{k}=\mathbf{1}$ & $\boldsymbol{f}$ s & $\boldsymbol{k}=\mathbf{1}$ & $\boldsymbol{k}=\mathbf{2}$ \\
\hline Estimated frequencies (Hz) & & 41.62 & 45.80 & 49.97 & 54.14 & 58.31 \\
\hline \multirow{2}{*}{ Estimated Amplitudes } & (dB) & -38.75 & -21.14 & 16.46 & -22.23 & -41.88 \\
\cline { 2 - 7 } & (A) & 0.0164 & 0.1239 & 9.41 & 0.1094 & 0.0114 \\
\hline
\end{tabular}

From Table V and Figs. $7 a$ and $b$, it is noted that the SVD-Kalman technique allows identifying the fault signature with more clarity on the specified frequency band. This shows the merits of this technique. Furthermore, it is also observed that the magnitudes of the different harmonics increase with the number of the broken bar. This result is important for monitoring the severity of the fault.

Finally, very important remarks can be deduced from all the experimental tests conducted:

- The presence of a slight difference between both the theoretical frequencies (Table I) and those estimated (Tables II-IV-V) can be explained by the errors resulting from the speed measurement.

- The appearance of the components around the fundamental is a sign of the existence of rotor bars fault when the load is not variable.

- The harmonics magnitudes around the fundamental, associated with the fault, increase with the number of the broken bar. This indicates the level of the fault.

\section{CONCLUSION}

In this paper, a new reliable and effective SVD-Kalman approach for rotor fault diagnosis of the induction motor is proposed in order to enhance simultaneously:

The frequency resolution: allowing a better distinction between harmonics that are close to each other at a reduced acquisition time. 
The magnitudes estimation of these harmonics: enabling better monitoring of the severity of the searched faults.

The reduction of the computation time.

This approach is based on the combination of two algorithms. The first consists in the decomposition into singular values of the estimated covariance matrix from the stator current in order to improve the frequency resolution of the stator current spectrum. The second allows the estimation of these signatures magnitudes using the KF in order to improve the estimation of the magnitudes of these signatures.

Moreover, the identification of the fault using the combination of both algorithms is carried out on a limited frequency band where the fault signature is likely to appear. This solution leads to the reduction of the computation time without affecting the efficiency of the analysis. Finally, the results obtained experimentally show the effectiveness and merits of the proposed approach compared to the conventional Periodogram method to identify the frequency signatures.

\section{REFERENCES}

[1] P. Karvelis, G. Georgoulas, I.P. Tsoumas, J.A. Antonino-Daviu, V. Climente-Alarcón, and C.D. Stylios, "ASymbolic Representation Approach for the Diagnosis of Broken Rotor Bars in Induction Motors", IEEE on Industrial Informatics, Vol 11 No.5, pp. 1028 - 1037, 2015.

[2] A. Bendiabdellah, A.H. Boudinar, N. Benouzza, and M. Khodja, "The enhancements of broken bar fault detection in induction motors ", In IEEE of the International Conference on Electrical Machines and Power Electronics, International Conference on Optimization of Electrical \& Electronic Equipment and International Symposium on Advanced Electromechanical Motion Systems , Side., Turkey, pp. 81-86, 2015

[3] A. Stefani, A. Bellini, and F. Filippetti, "Diagnosis of Induction machines'Rotor Faults in Time-Varying Conditions", IEEE Transaction on Industrial Electronics, Vol. 56 No. 11, pp. 4548-4556, 2009

[4] S. Toscani, M. Faifer, M. Rossi, L. Cristaldi, and M. Lazzaroni, "Effects of the Speed Loop on the Diagnosis of Rotor Faults in Induction Machines ", IEEE Transactions on Instrumentation And Measurement, Vol. 61 No. 10, pp.27132722,2012

[5] S.H. Kia, H. Henao, and G.A Capolino, "Zoom-MUSIC frequency estimation method for three phase induction machine fault detection", in IEEE Annual Conference of Industrial Electronics Society, pp.2603-2608, 2005.

[6] A. C. Hernandez, J. A. Daviu, J. P. Llinares, V. C. Alarcon and V. F. Galiana, "Transient-Based Rotor Cage Assessment in Induction Motors Operating With Soft Starters," IEEE Transactions on Industry Applications, vol. 51, No. 5, pp. 3734-3742, Sept.-Oct. 2015.

[7] F.A. Aimer, A.H. Boudinar, and A. Bendiabdellah, " Asynchronous Motor Rotor Faults Detection and Location using the Short Time Fourier Transform Approach", Journal of Electrical Engineering, Vol. 14, No. 18,pp.1-5, 2014.

[8] E. H. Bouchikhi, V. Choqueuse, M. E. H. Benbouzid, J. F. Charpentier and G. Barakat, "A comparative study of timefrequency representations for fault detection in wind turbine," IECON 2011 - 37th Annual Conference of the IEEE Industrial Electronics Society, Melbourne, VIC, , pp. 3584-3589,2011.

[9] H. Henao, G.A. Capolino, M. Fernández-Cabanas, F. Filippetti, C. Bruzzese, E. Strangas, R. Pusca, J. Estima, M. RieraGuasp, and S.H. Kia, "Trends in Fault Diagnosis for Electrical Machines", IEEE Ind. Electron. Magazine, Vol. 8 No. 2 , pp. 31-42, 2014. 
[10] Y.S. Park, L.R. Hochberg, E.N. Eskandar, S.Cash Sydney, W. Truccolo, "Adaptive parametric spectral estimation with Kalman smoothing for online early seizure detection", In IEEE/EMBS $20136^{\text {th }}$ Conference on Neural Engineering, San Diego, CA, pp. 1410-413, 2013

[11] E.H. El Bouchikhi, V. Choqueuse, and M.E.H. Benbouzid, "Induction Machine Faults Detection using Stator Current Parametric Spectral Estimation", Mechanical Systems and Signal Processing, Vol. 52-53, pp 447-464, 2015.

[12] K. Yahia, M. Sahraoui, A. J. M. Cardoso and A. Ghoggal, "The Use of a Modified Prony's Method to Detect the Airgap-Eccentricity Occurrence in Induction Motors," IEEE Transactions on Industry Applications, vol. 52, No. 5, pp. 3869-3877, 2016.

[13] D. Morinigo-Sotelo, R. J. Romero-Troncoso, J. A. Antonino-Daviu and K. N. Gyftakis, "Reliable detection of broken rotor bars in induction motors via MUSIC and ZSC methods," 2016 XXII International Conference on Electrical Machines (ICEM), Lausanne, pp. 2881-2886, 2016.

[14] B. Xu, L. Sun, L. Xu and G. Xu, "An ESPRIT-SAA-Based Detection Method for Broken Rotor Bar Fault in Induction Motors," IEEE Transactions on Energy Conversion, vol. 27, no. 3, pp. 654-660, 2012.

[15] A.H. Boudinar, N. Benouzza, A. Bendiabdellah, and M.E.A. Khodja, "Induction Motor Bearing Fault Analysis Using a Root-MUSIC Method ", IEEE Transactions on Industry Applications, Vol. 52 No.5, pp. 3851-3860,2016.

[16] Y. Trachi, E. Elbouchikhi, V. Choqueuse and M. E. H. Benbouzid, "Induction Machines Fault Detection Based on Subspace Spectral Estimation," IEEE Transactions on Industrial Electronics, vol. 63, No. 9, pp. 5641-5651, 2016.

[17] M. Sahraoui, A.J. Marques Cardoso, and A. Ghoggal, "The Use of a Modified Prony's Method to Track the Broken Rotor Bars Characteristic Frequencies and Amplitudes, in Three-Phase Induction Motors", IEEE Transactions on Industry Applications, Vol. 51 No. 3, pp. 2136-2147, 2015.

[18] A. Toloei, S. Niazi, "State Estimation for Target Tracking Problems with Nonlinear Kalman Filter Algorithms, " International Journal of Computer Applications, Vol. 98, No.1, pp. 30-36, 2014.

[19] C.K. Chui, G. Chen, Kalman Filtering with Real-Time Applications, Springer, Berlin, 2009.

[20] T. Ameid, A. Menacer, H. Talhaoui,and I. Harzelli, "Rotor resistance estimation using Extended Kalman filter and spectral analysis for rotor bar fault diagnosis of sensorless vector control induction motor," Measurement(ELSEVIER), Vol.111, ,pp. 243-259, 2017.

[21] F. Alonge, F. D'Ippolito and A. Sferlazza, "Sensorless Control of Induction-Motor Drive Based on Robust Kalman Filter and Adaptive Speed Estimation," IEEE Transactions on Industrial Electronics, vol. 61, No. 3, pp. 1444-1453, 2014.

[22] Y.H. Kim, Y.W. Youn, D.H. Hwang, J.H. Sun, and D.S. Kang, "High-Resolution Parameter Estimation Method to Identify Broken Rotor Bar Faults in Induction Motors", IEEE Transactions on Industrial Electronics, Vol. 60, No. 9, pp.4103-4117, 2013

[23] J.H. Jung, J.J. Lee, and B.H. Kwon, "Online Diagnosis of Induction Motors Using MCSA", IEEE Transactions on Industrial Electronics, Vol. 53 No.6, pp.1842-1852, 2006.

[24] S. Chakkor, M. Baghouri, and A. Hajraoui, "Performance Analysis of Faults Detection in Wind Turbine Generator Based on High-Resolution Frequency Estimation Method", International Journal of Advanced Computer Science and Applications,Vol. 5 No.4, pp.139-148, 2014.

[25] H. Jiang, J. Chen, G. Dong, T. Liu, and G. Chen, "Study on Hankel matrix-based SVD and its application in rolling element bearing fault diagnosis", Mechanical Systems and Signal Processing, Vol. 52-53, pp. 338-359, 2015.

[26] F. Auger, M. Hilairet, J.M. Guerrero, E. Monmasson, T. Orlowska-Kowalska, and S. Katsura, "Industrial applications of the Kalman filter A Review", IEEE Transactions on Industrial Electronics, Vol. 60 No.12, pp. 5458-5471, 2013. 